\title{
MODELO AMERICANO Y MODELO EUROPEO DE JUSTICIA CONSTITUCIONAL
}

\author{
Alfonso Ruiz Miguel \\ Universidad Autónoma de Madrid
}

\section{Introducción(*)}

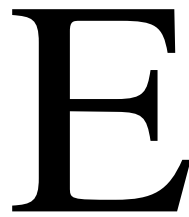

n este escrito pretendo poner en cuestión algunas distinciones tradicionalmente consagradas en la comparación entre los dos modelos clásicos de justicia constitucional, el americano de la judicial review y el europeo de origen kelseniano. Aunque la convergencia entre ambos modelos ha sido reconocida ya hace tiempo desde el punto de vista de la ciencia política ${ }^{1}$, tengo la impresión de que la tendencia tradicional a destacar más los contrastes que las similitudes entre ambos sigue prevaleciendo en la cultura jurídico-constitucional española, con la que las páginas que siguen pretenden dialogar².

Para plantear analíticamente la comparación ordenaré la exposición en torno a lo que probablemente sean los tres criterios más señalados con los que suele identificarse el concepto de «jurisdicción», esto es, la caracterización de la función judicial, primero, como procedimiento de aplicación del Derecho frente a la creación jurídica; segundo, por la función de reso-

(*) Reelaboración del texto preparado para la participación en una mesa redonda en el V Congreso Hispano-Italiano de Teoría del Derecho, celebrado en Alicante los días 21 a 23 de octubre de 1999.

${ }^{1}$ Ya en 1988 el politólogo Brünneck afirmaba que «el grado de coincidencia entre los distintos métodos de revisión constitucional ha aumentado en los últimos decenios. Este proceso probablemente ha sido promovido por los frecuentes encuentros bilaterales y multilaterales entre magistrados constitucionales. La razón decisiva para el aumento de la similitud en las estructuras organizativas de los tribunales constitucionales reside en el hecho de que cumplen eminentemente las mismas funciones en todos los países aquí investigados» (Brünneck 1988, p. 233). Más recientemente, el constitucionalista alemán Peter Häberle ha destacado la importancia que para la integración europea y el desarrollo de un ius commune constitutionale tienen, junto a otros factores, las conferencias que celebran los tribunales constitucionales europeos (cf. Häberle 1996, pp. 198-9).

${ }^{2}$ Una apreciación similar, extendida a la doctrina constitucional europea, en Aja y González Beilfuss 1998, pp. 258-9. 
lución de controversias de carácter concreto; y, tercero, por la situación de independencia e imparcialidad de sus órganos y decisiones. Analizar el juego de esos criterios en los dos modelos mencionados creo que permitirá revisar también algunos problemas de la justicia constitucional relevantes desde el punto de vista de la filosofía del Derecho, y tanto en su vertiente teórico-jurídica como filosófico-política. En esta indagación, naturalmente, la reflexión en torno a los tres criterios mencionados es sobre todo una especie de pretexto para plantear tales problemas, entre los que excluyo la pretensión de buscar, y menos de encontrar, esencia alguna de la función judicial $^{3}$.

\section{Aplicación y creación de Derecho}

El primer criterio anunciado, el de que la actividad judicial -como también la administrativa- es eminentemente un procedimiento de aplicación y no de creación del Derecho, está estrechamente relacionado con el diseño democrático-liberal de la división de los poderes estatales, en el que sólo al legislativo, como representante de la voluntad popular, le cumple la función de crear Derecho. Con independencia de la distancia entre este diseño ideal y la realidad de la actividad judicial ordinaria, es en la justicia constitucional donde resulta más ostensible la actividad creadora de Derecho ${ }^{4}$.

En efecto, en las decisiones sobre la constitucionalidad de las leyes se pueden observar fácilmente los tres significados básicos en los que los jueces pueden «crear Derecho»: en primer lugar, desde un punto de vista puramente formal, claramente destacado por Kelsen, porque toda sentencia introduce una nueva norma en el sistema (es la norma individual que decide el caso, en cuanto enunciado diferente de otros, con independencia de su contenido significativo); en segundo lugar, desde el punto de vista del contenido, porque al menos algunas sentencias no apliquen mecánicamente el

\footnotetext{
${ }^{3}$ En efecto, no creo que ninguno de los tres criterios, u otros alegables, sea «esencial» a la función judicial, en el sentido de que pueda encontrarse en todos los casos y sólo en el ámbito de lo que suele y puede considerarse jurisdiccional, que al fin al cabo es un concepto polisémico y sobre el que toda definición útil ha de ser estipulativa. Así, además de los tres criterios citados, ni el carácter contradictorio del proceso, ni la motivación de las decisiones, ni el carácter de árbitro o tercero del juez son rasgos universales de la función judicial, sea porque han existido y existen actividades distintas y aun opuestas a las anteriores que se han considerado judiciales, sea porque actividades distintas a las judiciales también pueden caracterizarse mediante alguno de tales rasgos.

${ }^{4}$ Con cierta exageración, y no sin alguna falta de sensibilidad tanto hacia el realismo jurídico y a la tradición teórica kelseniana como hacia el papel creativo de los jueces ordinarios, se ha llegado a decir que una de las consecuencias del control de constitucionalidad «es que todo acto de aplicación del Derecho es en alguna medida un acto de creación de Derecho. La otra es que la jurisdicción constitucional no es lo opuesto a la política, sino que hasta cierto punto es política» (Grimm 1988, p. 169).
} 
Derecho preexistente sino que lo innoven, bien mínimamente en la medida en que deban elegir entre alguno de los criterios de los que las normas aplicables son marcos abiertos a distintas posibilidades (que es también una idea kelseniana, conectada con su visión del doble carácter de creación y aplicación en el proceso de concreción de los grados inferiores del ordenamiento jurídico), bien incluso, en la forma fuerte de la creación material, porque establezcan ex novo criterios que no se reconocieran como existentes previamente; en tercer lugar, en fin, desde el punto de vista de los efectos, porque el criterio adoptado en la sentencia, con independencia de su contenido más o menos repetitivo o innovador respecto de lo que las normas previas establezcan, reciba en el sistema no sólo eficacia para el caso resuelto sino también fuerza erga omnes.

Pues bien, donde puede haber diferencias entre los dos modelos de justicia constitucional, el americano y el kelseniano, no es en el primer significado mencionado de la idea de creación judicial, que es puramente formal y dependiente de la convención adoptada sobre el uso del término «norma», sino en los otros dos. Y, en particular, por un lado, dentro del segundo significado, en la forma fuerte de la creación desde el punto de vista del contenido, cuyo comentario voy a dejar pendiente hasta la discusión del punto siguiente, y, por otro lado, en el tercer significado apuntado, bajo el punto de vista de los efectos erga omnes, al que me referiré ahora.

La visión más extendida en este último punto viene a diferenciar entre el sistema americano y el europeo de la siguiente manera: mientras en el sistema americano la fuerza erga omnes se produce en toda sentencia del Tribunal Supremo (al igual que en las de cualquier otro tribunal, respecto de los inferiores) como derivación del valor de precedente que su justificación adquiere para ulteriores casos similares, en cambio, en el sistema austriaco, la fuerza erga omnes se predica únicamente, como mero efecto de la cosa juzgada y, con independencia de los fundamentos, sólo del fallo de las sentencias declaratorias de la inconstitucionalidad de una ley, esto es, no en las declaratorias de la constitucionalidad ni, en la variante germana y española, en la resolución de recursos individuales en amparo de derechos fundamentales 5 .

\footnotetext{
${ }^{5}$ La tesis es formulada así por Favoreu, para quien lo que cuenta para hablar de jurisdicción constitucional es que «el Tribunal Constitucional diga el derecho con autoridad de cosa juzgada y que sus declaraciones de inconstitucionalidad puedan desembocar en anulaciones con efecto erga omnes», a diferencia, añade, del mero reenvío al parlamento para una reconsideración, donde hay consejo más que jurisdicción, o de la reescritura y sustitución positiva de la ley, donde hay más legislación que jurisdicción (cf.Favoreu 1986, p. 23).
} 
En su origen, es muy verosímil que la ausencia del sistema de stare decisis hiciera casi impensable la introducción en la Europa continental de un sistema de control difuso de constitucionalidad, que habría generado una gran inseguridad jurídica ${ }^{6}$. Y de ahí no sólo la concentración del control de constitucionalidad en un tribunal diferenciado y especializado, sino también la consideración de éste por Kelsen, su primer defensor y teorizador, como legislador negativo. En efecto, es plenamente significativo en este aspecto que en su famoso artículo de 1928 en defensa de una garantía jurisdiccional de la Constitución, Kelsen negara un tanto paradójicamente que un Tribunal Constitucional al modo austriaco ejerciera «verdaderamente una función jurisdiccional». Y lo negó como resultado de la convergencia de dos ideas: de un lado, su caracterización de la diferencia de tal función respecto de la legislativa «ante todo en que ésta crea normas generales, mientras que aquélla sólo crea normas individuales» $\mathrm{y}$, de otro lado, su visión del control de constitucionalidad efectivo como anulación de normas; la suma de ambas ideas, por citar su conclusión literalmente, daba por resultado que «anular una ley es dictar una norma general [...no siendo] por así decirlo, sino producción con un signo negativo y, por tanto, una función legislativa» ${ }^{7}$. En cierto contraste con todo ello, reforzando la ambigüedad de sus tesis, Kelsen pudo considerar compatible esta configuración con su defensa de la naturaleza judicial del Tribunal Constitucional, en el sentido de independiente e imparcial, porque creía que la anulación de leyes, a diferencia de la legislación positiva, era, desde un punto de vista sustantivo, aplicación del Derecho y apenas libre creación, hasta el punto de afirmar que aquélla se encuentra «absolutamente determinada por la Constitución» ${ }^{8}$, también -no se puede dejar de recordarlo- porque en su diseño quedaba tajantemente excluida la revisión constitucional relativa a principios generales de justicia, libertad, igualdad y moralidad.

\footnotetext{
${ }^{6} \mathrm{Cf}$. von Beyme 1988, p. 30.

${ }^{7}$ Cf. Kelsen 1928, p. 130.

${ }^{8}$ Explica Kelsen: «Aparece en este punto la distinción entre la elaboración y la simple anulación de las leyes. La anulación de una ley se produce, esencialmente, en aplicación de las normas de la Constitución. Está ausente casi por completo aquí la libre creación que caracteriza a la legislación. Mientras que el legislador no está vinculado a la Constitución más que con respecto al procedimiento y solamente de forma excepcional respecto al contenido de las leyes que debe dictar -y tan sólo por medio de principios o directivas generales-, la actividad del legislador negativo, de la jurisdicción constitucional, está, por el contrario, absolutamente determinada por la Constitución. Y es precisamente por ello por lo que su función se asemeja a la de cualquier otro tribunal, pues se trata principalmente de aplicación y sólo en una débil medida de creación del Derecho y, por tanto, de algo propiamente jurisdiccional» (Kelsen 1928, p. 131).
} 
Ahora bien, en lo que se refiere a este criterio de la aplicación y la creación del Derecho, entre la propuesta kelseniana y el modelo actual de justicia constitucional en sistemas como el alemán, el español y, aunque quizá en menor medida, el italiano me parece que hay ya más diferencias que entre ese modelo y el americano. En efecto, por recoger brevemente fenómenos conocidos ${ }^{9}$, las sentencias interpretativas, que establecen significados constitucionalmente válidos e inválidos de un texto legal, han servido para introducir criterios positivos de constitucionalidad a través de sentencias no anulatorias cuyo alcance, además, no se restringe exclusivamente al fallo sino que remite a la argumentación previa; por su parte, las sentencias constructivas, que indican qué modificaciones necesita una ley para ser constitucional, han permitido controlar no sólo las acciones del legislador sino también, aunque en menor medida, algunas de sus omisiones; en fin, en el caso de los tribunales constitucionales alemán y español especialmente, a través de la resolución de los recursos individuales de queja o de amparo, la construcción de una rica y compleja doctrina sustantiva en materia de derechos y libertades ha tenido decisivos efectos en el conjunto del Derecho legislado y del aplicado por el conjunto de los jueces ${ }^{10}$. El resultado natural de este proceso ha sido la progresiva introducción en el sistema europeo de un sistema de precedente, en especial respecto de las decisiones de los tribunales constitucionales, cada vez menos diferenciable del propio del common $l a w^{11}$. El último y más completo eslabón en este proceso de consolidación del principio del stare decisis constitucional lo ofrece, me parece, el artículo 5.1 de la Ley Orgánica del Poder Judicial española, aprobada en 1985, que dice: «La Constitución es la norma suprema del ordenamiento jurídico, y vincula a todos los Jueces y Tribunales, quienes interpretarán y aplicarán las leyes y los Reglamentos según los preceptos y principios constitucionales, conforme a la interpretación de los mismos que resulte de las resoluciones dictadas por el Tribunal Constitucional en todo tipo de proce-

${ }^{9}$ Cf., por ejemplo, Rubio 1993, pp. 510ss; Jiménez Campo 1997a, pp. 45ss; y Aja y González Beilfuss 1998, pp.276-79.

${ }^{10}$ En tal medida, las decisiones constitucionales declarando esferas de libertad constitucionalmente garantizadas para el ciudadano (p.ej., el aborto en USA), incluidos los derechos procesales ante el Estado (el caso Miranda, la prohibición de usar pruebas ilícitas, las limitaciones a los análisis corporales, etc.) pueden actuar tanto como anulaciones o inaplicaciones de leyes -o de interpretaciones de leyes- cuanto como nuevos criterios positivos que «congelan» ulteriores regulaciones legales (que, en el caso español al menos, han solido respetar hasta limitarse a la mera reproducción de los criterios del Tribunal Constitucional).

${ }^{11}$ Dentro de una construcción tendente a destacar los rasgos específicos del modelo europeo respecto del americano, Pedro Cruz Villalón ha reconocido que el «resultado final [...] puede no ser muy distinto en uno y otro sistema, dada la virtualidad del principio «stare decisis»» (Cruz 1987, p. 35). Sobre el tema, en general, véase MacCormick y Summers 1997. 
sos»; y obsérvese que esta referencia final a «las resoluciones en todo tipo de procesos» parece conducir a considerar doctrina aplicable la de cualquier sentencia, sea favorable o contraria al acogimiento de la inconstitucionalidad, e incluyendo también las que resuelven recursos de amparo.

Es verdad que una diferencia que todavía subsiste en alguna medida entre el modelo americano y el europeo reside en el efecto de expulsión del ordenamiento jurídico de la norma declarada inconstitucional que se produce en el segundo, efecto que se relaciona con la mayor irreversibilidad de tal tipo de decisiones en ese modelo ${ }^{12}$. Ahora bien, esta diferencia, que es innegable en el plano formal, podría ser mucho más tenue en el plano de la realidad, y tanto porque en el modelo americano los criterios constitucionales consolidados por el Tribunal Supremo tienen de hecho una vinculatoriedad muy fuerte para todos los órganos del Estado, incluidos los legisladores $^{13}$, como porque en el sistema europeo no está tan claro, aunque es asunto discutido y abierto, que la expulsión de una norma por inconstitucional tenga o deba tener un carácter tan irreversible como para excluir la aprobación futura de una norma igual o similar ${ }^{14}$.

Las anteriores observaciones, que cuando menos tienden a limar las aristas de un agudo contraste entre el modelo americano y el europeo, tal vez permitan abordar bajo una nueva luz el problema de si los tribunales constitucionales europeos deberían poder seleccionar más libremente los recursos individuales sometibles a su decisión. Ante este problema, que se plantea más agudamente en países como Alemania o España cuyos tribunales constitucionales tienen una notable sobrecarga de ese tipo de recursos, creo que la respuesta puede ser netamente afirmativa ${ }^{15}$. La razón fundamental reside en que los teóricos peligros de que en caso de introducción de una especie de writ of certiorari en estos sistemas decaería gravemente la posi-

\footnotetext{
${ }^{12}$ Víctor Ferreres ha destacado como «gran diferencia» en este punto el que mientras en el modelo americano es posible, como proceso fisiológico, un cierto grado de desobediencia descentralizada a la jurisprudencia del Tribunal Supremo, en el europeo la desobediencia ante la decisión de inconstitucionalidad de una ley ha de concentrarse en el parlamento (cf. Ferreres 1997, pp. 205-6). Cabe precisar, en todo caso, que este último rasgo, en sistemas como el alemán o el español al menos, no afecta a la jurisprudencia en materia de amparo o a la que se deduce de recursos y cuestiones resueltos en favor de la constitucionalidad de las leyes, que puede contener criterios interpretativos que restringen las posibilidades de actuación del legislador.

${ }^{13}$ Cf. sobre ello Gunther 1991, p. 28. Por lo demás, ya en 1966 Cappelletti había precisado que en Estados Unidos el stare decisis produce en la práctica no una mera desaplicación para el caso sino que «termina por asumir una verdadera y propia eficacia erga omnes», convirtiéndose «en una verdadera y propia anulación de la ley», y añade que la posibilidad teórica de resurrección de una norma por overruling se ha usado en casos «realmente excepcionales» (Cappelletti 1966, p. 41-42).

${ }^{14}$ Cf. Ferreres 1997, pp. 199ss, así como Aja y González Beilfuss 1998, p. 284.

${ }^{15}$ Sobre ello, ver el documentado trabajo de López Pietsch 1998.
} 
bilidad de tutela efectiva en materia de derechos y libertades son tanto más lejanos cuanto más consolidado esté el actual proceso de respeto a los precedentes del Tribunal Constitucional. En efecto, una adecuada selección de casos permitiría, además de aclarar eventuales jurisprudencias oscilantes, resolver casos dudosos con criterios bien meditados y fundamentados, con un mayor y mejor efecto expansivo material en el conjunto de las decisiones judiciales. Ese efecto material, por lo demás, podría reforzarse procesalmente mediante la centralización de un recurso general en materia de derechos fundamentales, e incluso de garantías constitucionales, en una sala del Tribunal Supremo con la función específica de unificar jurisprudencia en aplicación de los criterios del Tribunal Constitucional.

\section{Abstracción y concreción en el control de constitucionalidad}

El segundo criterio de jurisdiccionalidad, la idea de que ejercer justicia consiste en resolver controversias y casos concretos, que estuvo en el centro de la polémica de Carl Schmitt contra la defensa kelseniana de la jurisdicción constitucional ${ }^{16}$, ha sido el otro foco principal de contraste entre el modelo de justicia constitucional americano y el europeo. El criterio de que resolver conflictos concretos -o, como decía Tocqueville, «pronunciarse sobre casos particulares», afectando «a un interés individual» ${ }^{17}$ - es esencial al poder judicial, presenta en abierto contraste el modelo americano, de justicia constitucional incidenter, donde un particular alega el perjuicio de un acto estatal concreto contrario a sus derechos constitucionales, y el modelo europeo, de justicia constitucional principaliter, donde una minoría parlamentaria o algún órgano con especial legitimación alegan directamente que se ha dictado una ley en violación de la Constitución y el examen subsiguiente tendría el carácter de un control abstracto, alegadamente del todo diferente al juicio de un caso concreto ${ }^{18}$. Como es sabido, ya el desarrollo de este modelo en varias constituciones europeas ${ }^{19}$ introdujo dos mecanis-

${ }^{16}$ Según Schmitt, «una sentencia judicial es [...] la decisión de un "caso" "sobre la base de una ley"» (Schmitt 1931, p. 79), mientras que «[1]a aplicación de una norma a otra norma es algo cualitativamente distinto de la aplicación de una norma a un contenido real» (quiere decir, a un caso concreto) (ib., p. 84), pues «compáranse entre sí reglas generales, pero no se subsume ni se 'aplica' una a otra» (ib., p. 85).

${ }^{17}$ De la démocratie en Amérique, libro I, cap. 6 (en la edición castellana de Eduardo Nolla, La democracia en América, Madrid, Aguilar, 1988, Tomo I, pp. 101 y 105); por su parte, también Montesquieu había identificado el «poder de juzgar» con aquel por el que el magistrado «castiga los crímenes o juzga las disputas entre los particulares» (De l'esprit des lois, XI, VI).

${ }^{18} \mathrm{~A}$ la distinción anterior se suele superponer otra diferente, de tipo organizativo: la distinción entre justicia difusa y concentrada, que alude a los órganos encargados del control, según sean todos los tribunales ordinarios o exclusivamente un tribunal único, normalmente especializado.

${ }^{19}$ Así, en las de Austria, Alemania, Italia, España, Bélgica y Portugal. 
mos de jurisdicción incidental: en primer lugar, la cuestión judicial de inconstitucionalidad, que un juez ordinario puede presentar ante el Tribunal Constitucional cuando tiene dudas a propósito de una ley aplicable al caso, control que, se ha dicho ${ }^{20}$, si bien es concreto en su planteamiento inicial por el juez, es en cambio abstracto en su modo de resolución por el Tribunal Constitucional; y, en segundo lugar, los recursos de queja o de amparo al modo alemán o español ${ }^{21}$, que permiten a cualquier ciudadano acudir al Tribunal Constitucional por la aplicación de algún acto estatal contrario a la Constitución y que, tras un determinado procedimiento, pueden llegar a dar lugar a una declaración abstracta de inconstitucionalidad de una ley.

Con independencia de esos desarrollos, el contraste entre esa forma abstracta y la forma concreta o caso por caso, al estilo americano, ha sido presentado como una contraposición tajante, con aristas muy netas, si bien en sentidos opuestos. Así, de un lado, como se mencionó hace poco, el criterio de Kelsen era que la forma abstracta del control de constitucionalidad, mediante el contraste directo entre el texto de la Constitución y el de la ley, había de ser un acto eminentemente aplicativo desde un punto de vista material o del contenido. De otro lado, y justo en la dirección opuesta, Genaro Carrió recogió lo que puede haber llegado a ser una opinión bastante común entre los juristas, cuando frente al recurso de constitucionalidad europeo alababa la judicial review americana (también argentina) por ser un tipo de control al por menor, en el que el tribunal no operaría como un «superlegislador que desde una superinvestidura puede derogar una ley entera»y que, por ello, no comportaría «un juicio de valor de alcance amplio, divorciado de una apreciación cuidadosa de los numerosos tipos de situaciones fácticas distintas que esa norma procura reglar»; únicamente el control sobre «ciertos casos concretos mediante decisiones ceñidas a los hechos de éstos y sólo aplicables a ellos» obligaría -concluía Carrió- «a una responsable actividad estrictamente judicial», imparcial, objetiva y limitada a los hechos relevantes ${ }^{22}$.

${ }^{20}$ Cf. Sentencia del Tribunal Constitucional español 161/1997, FJ 2; así como Jiménez Campo 1992, p. 17, que remite a Rubio Llorente y Cruz Villalón (pero con mayores matices, cf. Jiménez Campos 1997b, p. 97-100).

${ }^{21}$ El recurso individual en la Constitución austriaca, introducido en 1975, se limita a la posibilidad de impugnar una ley inconstitucional aplicable sin la intervención judicial ni administrativa, por lo que su alcance es de mucho más limitada significación que en los dos casos citados en el texto (cf. Favoreu 1986, p. 42; y Welan 1988, pp. 67-8).

${ }^{22}$ Carrió 1991, pp. 156-7. En buena parte, estas ideas de Carrió pueden rastrearse hasta Tocqueville, que veía en el carácter concreto del control americano de constitucionalidad un contrapeso al peligro del «inmenso poder político» conferido allí a los jueces: «Si el juez hubiese podido atacar las leyes de una manera teórica y general, si hubiese podido tomar la iniciativa y censurar al legislador, hubiese entrado estrepitosamente en la escena política. 
Para evaluar estas tesis en contraste no es suficiente, me parece, destacar la importancia que en los sistemas europeos han adquirido procedimientos como los citados poco más arriba, que tienen origen en una controversia concreta, puesto que lo que está aquí en cuestión es la diferencia entre este tipo de procedimientos y los de carácter abstracto. En realidad, a la vista de la práctica, tiendo a pensar que la posición de Kelsen y la de Carrió yerran tanto en la contraposición tan tajante entre el control abstracto y el concreto como en la común suposición de que uno de ellos resulta tendencialmente menos materialmente creativo de Derecho que el otro, por donde termina resultado irrelevante la discrepancia entre ambos autores sobre cuál de las dos formas de control es más aplicativa o más creativa. Para hacer una comparación adecuada entre los dos sistemas conviene mantener distinguidos tres conceptos diferentes: el carácter incidental o principal del procedimiento, el tipo de argumentación y los efectos de la decisión. Los tres aparecen confundidos en los por lo demás claros textos de Carrió, cuando establece sendas correlaciones directas entre el tipo de procedimiento judicial y, de un lado, el tipo de argumentación y, de otro lado, el tipo de efectos de las decisiones judiciales. Sin embargo, las tres son cuestiones diferentes, y ambas correlaciones deben analizarse críticamente.

Así, por lo que afecta a la primera correlación, los razonamientos empleados por un tribunal pueden ser perfectamente del mismo tipo con independencia de que el procedimiento sea concreto y de carácter incidental o

Convertido en el campeón o adversario de un partido, hubiese llamado a tomar parte en la lucha a todas las pasiones que dividen el país. Pero cuando el juez ataca la ley en un debate oscuro y en una aplicación particular, oculta en parte a las miradas del público la importancia del ataque. Su sentencia únicamente tiene por objeto afectar a un interés individual, la ley sólo es herida por casualidad. Por otra parte, la ley así censurada no es destruida. Disminuye su fuerza moral, pero su efecto material no queda suspendido. Sólo poco a poco y bajo los golpes repetidos de la jurisprudencia, sucumbe finalmente. Además, se comprende con facilidad que al conferir al interés particular la iniciativa de la censura de las leyes, al ligar íntimamente el proceso hecho a la ley con el proceso hecho al hombre, se asegura que la legislación no será atacada a la ligera. En ese sistema no está expuesta ya a las agresiones diarias de los partidos. Al señalar las faltas del legislador se obedece a una necesidad real, se parte de un hecho positivo y apreciable, ya que debe servir de base a un proceso. [...]. Si el juez solamente pudiera atacar a los legisladores de frente, habría momentos en los que temería hacerlo y otros en que el espíritu de partido le empujaría a intentarlo cada día» (La democracia en América, I, p. 105).

En la línea de Carrió, aunque desarrollando un argumento algo diferente, apuntado por Klaus Günther y Habermas, Victor Ferreres ha defendido la mayor riqueza del debate público que tiende a producirse cuando la legitimación del recurso la tienen, directa o indirectamente, los particulares y no, como ocurre en el recurso de constitucionalidad europeo tipo, uno u otro órgano o grupo integrado en la organización política (cf. Ferreres 1997, pp. 176-8). Personalmente, sin embargo, no estoy tan seguro sobre la generalización presupuesta en este argumento sobre la mayor capacidad de los particulares sobre la de los distintos representantes parlamentarios para recoger los distintos argumentos relevantes. 
abstracto y de carácter principal. Por ejemplificarlo, no me parece que los razonamientos empleados por el Tribunal Supremo de Estados Unidos para resolver un caso concreto como el del castigo a una mujer por abortar (Roe v. Wade) tengan un carácter menos abstracto y universalista o estén más pegados a la «realidad concreta» -en la medida en que tal expresión no signifique que coincide más con la opinión que nos resulta más querida- que, póngamos por caso, los utilizados por las sentencias de los tribunales constitucionales alemán y español sobre la legislación despenalizadora del aborto. Creo que el grado de concreción o abstracción de un razonamiento judicial y su relación con los casos concretos no depende tanto del procedimiento adoptado como, junto con la mayor o menor profundidad o superficialidad del método argumentativo seguido, de lo que podría denominarse el grado de subsuntividad entre el caso o casos y la norma legal que los incluye y entre esta misma norma legal y el principio o norma constitucional tenido por relevante ${ }^{23}$. En tal sentido, por poner ejemplos claros, la relación de abstracción a concreción entre la norma de la Constitución española que atribuía solamente a los españoles los derechos de participación política excepto que una ley o tratado concediera el derecho de sufragio activo a no españoles y la norma del tratado de Maastrich que confiere también el derecho de sufragio pasivo a los ciudadanos de la Unión Europea residentes en España, que obligó a una reforma de la constitución tras una consulta al Tribunal Constitucional, es netamente más estrecha e inmediata que, por citar ahora ejemplos de una sentencia americana y otra española, entre la garantía constitucional contra la autoincriminación y el hecho de haber recibido la declaración voluntaria de un detenido sin haberle comunicado sus derechos (el caso Miranda v. Arizona) o entre el principio constitucional de la tutela judicial efectiva y el criterio que prohíbe utilizar pruebas obtenidas en violación de derechos fundamentales (la sentencia del Tribunal Constitucional español 114/1984).

En cuanto a la otra correlación implícita en la tesis de Carrió, entre el procedimiento y los efectos, ya he comentado que debe reconocerse la diferencia entre, de un lado, el sistema americano de mera inaplicación de las leyes inconstitucionales, que admite abiertamente la posibilidad de overruling o revisión en el futuro y la interpretación de su alcance como precedente (restricción a las rationes decidendi, distinguishing, etc.) y, de otro lado, el sistema austriaco de anulación definitiva y expresa de normas legales, que una vez declaradas inconstitucionales son, en el mejor de los casos, de

${ }^{23}$ Tal grado tiene que ver con la textura abierta del lenguaje y en la zona central es máximo, en la zona de penumbra decreciente y fuera de ella mínimo o nulo, según sea posible o no la interpretación extensiva o la analogía 
difícil restablecimiento. Sin embargo, esta diferencia no afecta necesariamente al grado de abstracción o generalidad de los efectos sobre los casos afectados, que es una función variable según las normas sometidas a examen constitucional con independencia de que el procedimiento sea incidental o principal. Por ejemplificarlo, la amplitud de los efectos de Roe v. Wade o de Brown v. Board of Education, a pesar de proceder de recursos en casos concretos, fue y ha seguido siendo tan inmensa, si no más, en su alcance como las declaraciones «abstractas» de inconstitucionalidad de un Tribunal Constitucional como el español anulando la norma que autorizaba la entrada policial en un domicilio en casos de flagrante delito sin autorización judicial o la que sometía a un tipo impositivo acumulativo las rentas de los cónyuges. Y si al hablar de los efectos se pensara no en la aplicabilidad a todos los casos similares al decidido, sino en el grado de relevancia o impacto social de éstas, todavía están por llegar en Europa decisiones tan decisivas como las que retrasaron el New Deal de Roosevelt o tan devastadoras como Dred Scott, cuya protección de la propiedad sobre los esclavos negros encendió la mecha de la guerra civil estadounidense.

Con todo, la conclusión general que de las anotaciones anteriores cabe extraer es que ni el sistema americano ni el europeo de control de constitucionalidad han terminado por realizar una tarea judicial típicamente aplicativa y de reducido alcance en la modificación del Derecho legislado. Una conclusión que, al confirmar con creces la elevada importancia política de la justicia constitucional, conduce directamente al tercero y último punto que pretendía plantear, relativo a la comparación de los sistemas de garantía de la independencia e imparcialidad de los jueces constitucionales en el modelo americano y el europeo.

\section{La independencia de los tribunales constitucionales}

El tercer y último criterio pendiente de comentario afecta a la situación de independencia e imparcialidad de los órganos y las decisiones en los modelos europeo y americano de justicia constitucional. Si se me permite dejar difuminadas algunas variaciones dentro de los distintos sistemas europeos, entre los elementos comunes de ambos modelos destacan, primero, el carácter del mecanismo de nombramiento de los jueces constitucionales, no burocrático-profesional sino electivo por parte de uno o varios órganos que, al menos en parte, están democráticamente legitimados de manera directa ${ }^{24}$,

\footnotetext{
${ }^{24}$ Digo «en parte» en referencia a la elección por cámaras parlamentarias, que en Europa, con excepciones como la de Francia y Austria, tienden a ser los órganos que mayor número de jueces nombran. Junto a ellos, también pueden tener una cierta capacidad de nombramiento, usualmente menor, los gobiernos y algunos órganos judiciales.
} 
y, segundo, su garantía de inamovilidad durante el período legalmente predeterminado. Cabe observar cómo uno y otro aspecto operan en sentido opuesto respecto del criterio de legitimación, pues si, en parte al menos, la elección produce una cierta legitimación democrática indirecta o derivada, en cierta medida similar a la que recibe el gobierno en un sistema parlamentario, la inamovilidad subsiguiente blinda a los jueces constitucionales respecto de prácticamente toda responsabilidad política y democrática.

Tras el anterior trasfondo básicamente común, las principales diferencias entre el modelo americano y el europeo pueden cifrarse en dos $^{25}$ : por una parte, la figura del nombramiento presidencial unido al procedimiento de advice and consent realizado mediante la audiencia del Senado en el primero, en contraposición al más o menos extenso sistema europeo de reparto de cuotas con capacidad de vetar nombres entre los partidos políticos con la suficiente capacidad parlamentaria ${ }^{26}$; y, por otra parte, el diferente esquema de inamovilidad, que en el caso americano procede del nombramiento vitalicio y, por tanto, de la imposibilidad ulterior de «castigo» a través de la libre remoción, mientras en el modelo europeo procede del nombramiento con un término preestablecido -bien un período de ejercicio de 6 a 12 años bien la llegada del juez a la edad de setenta años- y, por tanto, de la imposibilidad ulterior de «premio» a través de la reelección. De entre los varios temas que estas diferencias podrían sugerir seleccionaré dos: la primera anima a preguntar por el interés de introducir en el sistema europeo un mecanismo de audiencia pública en el parlamento y la segunda suscita la duda de cuál de los dos sistemas de terminación del cargo es preferible para el objetivo de la independencia de los jueces respecto de las influencias políticas.

Antes de intentar responder a una y otra cuestión aventuraría que entre las dos diferencias que he señalado existe una cierta correlación interna, en el sentido de que, tomando siempre este tipo de generalizaciones como lo que son, el nombramiento presidencial vitalicio con intervención del Sena-

\footnotetext{
${ }^{25}$ No son las únicas, pues es importante la diferencia sobre el momento y el contexto histórico en que uno y otro se establecen: mientras el control americano surge con influencias inglesas y iusnaturalistas, sin asomo de desconfianza hacia el poder judicial, el europeo es de influencia doctrinal positivista y se construye con una cierta desconfianza hacia los jueces; este último rasgo, por lo demás, ha sido perdurable, hasta el punto de que los tribunales constitucionales, sociológicamente, expresan no el poder de los jueces, sino más bien de los juristas teóricos, un ProfessorenRecht (este rasgo puede explicar una de las razones por las que, en España al menos, los juristas teóricos favorecen la existencia del Tribunal Constitucional, pues, frente a una tradición de relativamente escasa o lenta influencia en el Tribunal Supremo, aquél está formado principalmente por profesores y es un medio mucho más receptivo a su influencia y todavía más decisivo en la organización jurídica.

${ }^{26}$ En Austria es la costumbre y no la necesidad la que impone una práctica de pacto entre los dos partidos que han gobernado en coalición.
} 
do americano ha tendido a producir tribunales disociados de las mayorías democráticas sucesivas en mayor medida que en el caso de los tribunales europeos, a lo que probablemente han contribuido tanto la menor frecuencia de las renovaciones parciales en el caso americano como lo que podría denominarse la mayor sintonía media obtenida por la elección parlamentaria por mayorías reforzadas en el caso europeo. Si esto es así, en Europa tendería a ser más difícil que en Estados Unidos la elección de magistrados ideológicamente muy sesgados y caracterizados. Una circunstancia, por cierto, que en este último país se ha producido con notable insistencia a pesar de las audiencias del Senado, que al fin y al cabo termina decidiendo por simple mayoría.

Aun así, la correlación interna que he aventurado no propone una incompatibilidad de principio entre el sistema europeo y la figura de la audiencia parlamentaria, especialmente respecto de los magistrados nombrados mediante elección parlamentaria, que sin duda podrían ser sometidos a dicha audiencia ante una comisión antes de su elección por la cámara correspondiente. El procedimiento parece de articulación constitucional mucho menos fluida, en cambio, para los casos en los que en línea de principio más razonable sería introducir controles externos y democráticos, esto es, para los magistrados designados por órganos distintos al parlamento. Junto a ello, y por echar algunos granos de sal sobre las virtudes democráticas del sistema de audiencia, tal vez no sea ocioso tener cautela ante sus posibles defectos en un país como el nuestro, como la probable división de los medios de comunicación y el consiguiente exceso de politización de las designaciones, el riesgo de mengua de la calidad de los candidatos tras la disminución de la cantidad de los dispuestos a sufrir eventuales calvarios públicos y, en fin, la posible depreciación del cargo de magistrado constitucional y, quizá, hasta el deterioro de las buenas prácticas del propio tribunal. Por lo demás, si considero el balance positivo del sistema en el caso del Tribunal Constitucional español, no me parece que haya especiales razones para que precisamente nosotros, que no estamos entre los más viejos del lugar en la práctica democrática, debamos iniciar los experimentos en este punto.

Me falta comentar, para concluir ya, el tema de la relación entre la independencia y las condiciones de terminación del cargo de juez constitucional, sobre el que sólo quiero esbozar un par de ideas. A primera vista, la independencia está más y mejor garantizada en el modelo americano de jueces vitalicios que en el europeo de exclusión de la renovación en el cargo tras el período preestablecido. El mayor defecto de este segundo sistema parece residir en que el «premio» sigue siendo posible, en especial por la eventual aparición de una práctica de nombramientos para otros cargos 
prestigiosos. Frente a esta posibilidad, que puede haberse verificado en alguna medida en la pedestre realidad, se ha argumentado que el peso decisivo para la independencia de los jueces constitucionales reside en la cultura interna de este tipo de tribunales, más basada en el empleo de técnicas jurídicas que en reglas de la práctica política ${ }^{27}$.

Es verdad que este tipo de respuestas puede resultar poco satisfactorio para quien crea que es inevitable, absolutamente inevitable, la posesión de una ideología y que, aunque ciertamente con límites, es difícilmente evitable que tal ideología no aliente detrás de la técnica jurídica en las más importantes decisiones que adoptan los jueces constitucionales. $\mathrm{Y}$ en tal nivel de exigencia, para el juicio global de la institución es indiferente que esa humana deficiencia -que, por supuesto, es uno de los reparos sólidos de la objeción democrática al control judicial de constitucionalidad-, provenga de la lealtad desinteresada hacia una ideología o de la motivaciones asociadas a «premios» o «castigos» por este o aquel partido en el gobierno. Lo que, junto a una elemental observación comparativa de la realidad, conduciría a la consideración de que tampoco el sistema americano, como por lo demás ningún sistema, puede proporcionar una independencia judicial que esté más allá del bien y del mal. De tomar consideraciones como ésta hasta sus últimas consecuencias, sería la propia justicia constitucional la que saldría mal parada, y tanto en el modelo americano como en el europeo. Pero para quienes consideran razonable y beneficiosa la existencia del control de constitucionalidad, entre la fantasiosa independencia absoluta y la sumisión ciega ante una ideología partidista seguramente hay un espacio para una forma de independencia humanamente razonable, basada en el esfuerzo por el rigor, la profundidad y la calidad de las argumentaciones constitucionales, entre las que debe incluirse la buena filosofía del Derecho. Y en ese tipo de esfuerzo, siempre mejorable, me parece que, en conjunto y una vez más, entre el modelo americano y el europeo las diferencias no son cualitativas.

\section{Bibliografía}

Aja, Eliseo y González Beilfuss, 1998: «Conclusiones generales», en Aja, Eliseo (comp.), 1998: Las tensiones entre el Tribunal Constitucional y el Legislador en la Europa actual, Barcelona, Ariel, 1998.

Beyme, Klaus von, 1988: «The Genesis of Constitutional Review in Parliamentary Systems», en Lanfried 1988a, pp. 21-38.

Bouzat, Gabriel, 1991: «El control constitucional. Un estudio comparativo», en Nino 1991a, pp. 67-93.

\footnotetext{
${ }^{27}$ Cf. Pizzorusso 1988, pp. 113-4 y 125.
} 
Brünneck, Alexander von, 1988: «Constitutional Review and Legislation in Western Democracies», en Lanfried 1988a, pp. 219-60.

Cappelletti, Mauro, 1966: El control judicial de la constitucionalidad de las leyes en el Derecho comparado, México, Universidad Nacional Autónoma de México.

Carrió, Genaro R., 1991: «Una defensa condicionada de la judicial review (Sobre el método de control de constitucionalidad vigente en la Argentina)», en Nino 1991a, pp. 139-70.

Cruz Villalón, Pedro, 1987: La formación del sistema europeo de control de constitucionalidad (1918-1939), Madrid, Centro de Estudios Constitucionales.

Cruz Villalón, Pedro, et al., 1992: Los procesos constitucionales. Segundo Simposio de Derecho Constitucional (Sevilla, 27 y 28 de septiembre de 1991), Madrid : Centro de Estudios Constitucionales.

Favoreu, Louis, 1986: Les cours constitutionnelles, París, PUF.

Ferreres, Víctor, 1997: Justicia constitucional y democracia, Madrid, CEC.

Grimm, Dieter: «Comment», en Landfried 1988a, pp. 169-71.

Gunther, Gerald, 1991: Constitutional Law. Cases and Materials, 12 ${ }^{\mathrm{a}}$ ed., WestburyN.Y., The Foundation Press.

Häberle, Peter, 1996: «Derecho constitucional común europeo», en Pérez Luño, Antonio-Enrique (comp.), Derechos humanos y constitucionalismo ante el tercer milenio, Madrid, Marcial Pons, pp. 187-223.

Jiménez Campo, Javier, 1992: «Los procedimientos de declaración de inconstitucionalidad», en Cruz Villalón 1992, pp. 13-38.

Jiménez Campo, Javier, 1997a: «Qué hacer con la ley inconstitucional», La sentencia sobre la constitucionalidad de la Ley. Actas de las II Jornadas de la Asociación de Letrados del Tribunal Constitucional, Madrid, Tribunal Constitucional-Centro de Estudios Constitucionales, pp. 15-79.

Jiménez Campo, Javier, 1997b: «Algunos rasgos de la cuestión de inconstitucionalidad en España», en Ruiz-Rico 1997, pp. 81-109.

Kelsen, Hans, 1928: Wesen und Entwicklung der Staatsgerichtsbarkeit; trad. francesa, «La garantie jurisdictionnelle de la Constitution (La justicie constitutionnelle)», en Revue de Droit Publique et de la Science Politique en France et à l'étranger, XXXV, 1928; t. c. de Juan Ruiz Manero «La garantía jurisdiccional de la Constitución (la justicia constitucional)», en Escritos sobre la democracia y el socialismo, Madrid, 1988, pp. 109-55.

Kelsen, Hans, 1931: Wer soll der Hüter der Verfassung sein?; t. c. de Roberto J. Brie, supervisión de Eugenio Bulygin, ¿Quién debe ser el defensor de la Constitución?, Madrid, Tecnos, 1995.

Landfried, Christine (comp.), 1988a: Constitutional Review and Legislation: An International Comparison, Baden-Baden, Nomos Verlagsgesellschaft, 1988.

Landfried, Christine, 1988b: «Introduction» a Landfried 1988a, pp. 7-20.

Landfried, Christine, 1988c: «Constitutional Review and Legislation in the Federal Republic of Germany», en Landfried 1988a, pp. 147-67.

López Guerra, Luis, 1997: «Jurisdicción ordinaria y jurisdicción constitucional», en Ruiz-Rico 1997, pp.27-59. 
López Pietsch, Pablo, 1998: «Objetivar el recurso de amparo: las recomendaciones de la Comision Benda y el debate español», Revista Española de Derecho Constitucional, n. 53, mayo-agosto, pp. 115-51.

MacCormick, D. Neil, y Summers, Robert S. (comps.), 1997: Interpreting Precedents. A Comparative Study, Aldershot etc., Ashgate-Dartmouth.

Nino, Carlos S. et al., 1991a: Fundamentos y alcances del control judicial de constitucionalidad, Madrid, Centro de Estudios Constitucionales, 1991.

Nino, Carlos S., 1991b: «Los fundamentos del control judicial de constitucionalidad», en Nino 1991a, pp. 95-137.

Pizzorusso, Alessandro, 1988: «Constitutional Review and Legislation in Italy», en Landfried 1988a, pp. 109-26.

Rubio Llorente, Francisco, 1993: La forma del poder (Estudios sobre la Constitución), Madrid, Centro de Estudios Constitucionales.

Ruiz-Rico Ruiz, Gerardo (comp.), 1997: La aplicación jurisdiccional de la Constitución, Valencia, tirant lo blanch.

Schmidhauser, John R., 1988: «Judicial Activism and Congressional Responses in the United States», en Landfried 1988a, pp. 39-62.

Schmitt, Carl, 1931: Der Hüter der Verfassung; se cita por la t. c. de M. Sánchez Sarto, La defensa de la Constitución. Estudio acerca de las diversas especies y posibilidades de salvaguardia de la Constitución, Madrid, Tecnos, 1983.

Skouris, Wassilios, 1988: «Constitutional Disputes and Judicial Review in Greece», en Landfried 1988a, pp. 177-200.

Welan, Manfried, 1988: «Constitutional Review and Legislation in Austria», en Landfried 1988a, pp. 63-80. 